\title{
Healthcare-Associated Infection and Antimicrobial Resistance: Moving Beyond Description to Prevention
}

\author{
Bonnie L. Zell, MD, MS; Donald A. Goldmann, MD
}

The burden of healthcare-associated infection (HAI) and infections caused by healthcare-associated multidrug-resistant organisms (HA-MDROs) is substantial. The Centers for Disease Control and Prevention (CDC) estimates the annual number of HAIs in US hospitals at about 1.7 million, resulting in approximately 99,000 deaths, which makes HAI the most common infectious cause of death and one of the 10 leading causes of death overall.' Included in these troubling statistics are at least 350,000 infections and 12,000 deaths caused by methicillin-resistant Staphylococcus aureus (MRSA), vancomycin-resistant enterococci, and Clostridium difficile. It is estimated that infections caused by these multidrug-resistant pathogens result in at least $\$ 3.5$ billion in excess healthcare costs annually ( $\mathrm{CDC}$, unpublished data).

Three articles in this issue of the journal seek to shed additional light on estimates of the mortality, excess length of stay, and costs attributable to HAI..$^{2-4}$ The first of these, a study from France by Fabbro-Perray et al. ${ }^{2}$ measured the attributable mortality of HAI using a large, prospective, matched-cohort study design in a single hospital. The researchers determined that the population-attributable risk of HAI-attributable mortality throughout France lies between $2.1 \%$ and $4.0 \%$, on the basis of a $3 \%-6 \%$ range in the incidence of HAI in French hospitals. The second study, by Shurland et al., ${ }^{3}$ reports on a 9-year retrospective cohort study of $S$. aureus infection complicated by bacteremia in a single Veterans Health Administration system, found that patients with MRSA infection, excepting those with pneumonia, had higher mortality than did patients infected with methicillinsusceptible $S$. aureus. Together these 2 reports emphasize that HAI and HA-MDROs are associated with increased mortality independent of the patient's underlying illness. The third article, by Graves et al., ${ }^{4}$ is a prospective cohort study performed in 2 Australian hospitals that examined excess costs and length of stay attributable to HAI. ${ }^{4}$ The authors conclude that HAIs (urinary tract infections, lower respiratory-tract infections, and "other" infections) are associated with little excess cost or length of stay. Specifically, they found only US $\$ 17$ of extra variable costs and 2.58 extra hospital days per case of lower respiratory-tract infection, whereas urinary tract infection resulted in no excess costs or length of stay, and "other" infection resulted in no excess costs and only 2.61 extra hospital days. The findings of Graves et al. ${ }^{4}$ stand in marked contrast to previous higher estimates based on either a matched-cohort approach or the regression analysis methods favored by Fabbro-Perray et al. ${ }^{2}$ and Shurland et al. ${ }^{3}$

These 3 studies complement a vast and growing literature on the clinical and financial impact of HAI and HA-MDROs. Investigators have studied diverse populations and used a variety of epidemiological and statistical approaches to control for bias and confounding. Not surprisingly, the range of attributable harm and cost varies dramatically. For example, the cost per case of MRSA infection ranges from $\$ 7,000$ to $\$ 32,000$ in published studies. ${ }^{5}$ In general, but not always, the more aggressive the statistical approach to confounding, the lower the attributable risk. Of course, no amount of statistical manipulation can compensate fully for unmeasured factors, although the use of instrumental variables is a promising partial solution to this problem. ${ }^{6}$ At this point in the long history of infection control, it is reasonable to ask why we need more descriptive epidemiological studies of the attributable risk of HAI. Estimating the mortality, excess length of stay, and costs attributable to HAls would be an interesting academic exercise were there not increasing evidence that most, if not all, of these infections are preventable.

Although there are numerous examples of successful smallscale interventions to eliminate adverse events, including HAIs, in hospitals, the rate of overall improvement has been slow. The public is frustrated, and consumer groups are promoting legislation mandating public reporting of rates of infection and other healthcare-associated adverse events. Impatient with the pace of quality improvement nationally, in December 2004 the Institute for Healthcare Improvement (IHI) launched the 100,000 Lives Campaign, a national initiative to prevent 100,000 unnecessary inpatient deaths within 18 months. To help reach this goal, the Campaign proposed

Division of Healthcare Quality Promotion, Centers for Disease Control and Prevention, Atlanta, Georgia, and George W. Merck Fellow, the Institute for Healthcare Improvement, Cambridge, Massachusetts (B.L.Z.); and the Institute for Healthcare Improvement, Cambridge, Massachusetts (D.A.G.)

Received February 12, 2007; accepted February 12, 2007; electronically published February 20, 2007.

Infect Control Hosp Epidemiol 2007; 28:261-264

(C) 2007 by The Society for Healthcare Epidemiology of America. All rights reserved. 0899-823X/2007/2803-0002\$15.00. DOI: 10.1086/513722 
that hospitals implement 6 evidence-based interventions, 3 of which address the morbidity and mortality associated with infectious diseases: bloodstream infection (BSI), ventilatorassociated pneumonia, and surgical site infection. These interventions drew on evidence from epidemiological studies, as reflected in the guidelines issued by the Healthcare Infection Control Practices Advisory Committee (HICPAC) of the $\mathrm{CDC}$ and, in the case of ventilator-associated pneumonia, the American Thoracic Society and the Infectious Diseases Society of America. ${ }^{7,8}$ All US hospitals were invited to join, to acknowledge their involvement publicly, and to share their mortality results. More than 3,100 hospitals participated: $65 \%$ adopted the BSI intervention, $67 \%$ the ventilator-associated pneumonia intervention, and $72 \%$ the surgical site infection intervention. Notably, hospitals were urged to adopt the entire package of recommended practices, or "bundle," for each of these 3 interventions, and to measure success as "all-or-nothing." For example, "credit" for the BSI bundle required compliance with all components of the BSI bundle: compliance with hand hygiene, use of maximal barrier precautions, use of chlorhexidine skin antisepsis, optimal catheter site selection (with subclavian vein the preferred site for non-tunneled catheters), and daily review of the necessity for central lines, with prompt removal of unnecessary lines. The IHI estimates that participating hospitals prevented more than 122,000 needless deaths during the Campaign period (December 2004-June 2006). The 100,000 Lives Campaign did not attempt to determine to what extent the specific Campaign interventions-or the Campaign itself-contributed to the reduction in mortality that was observed.

Although hospitals that participated in the 100,000 Lives Campaign were not required to submit data regarding their compliance with the evidence-based processes of care for these 3 interventions, individual hospitals did report striking success in reducing infection rates. Examples of hospitals that significantly reduced rates of surgical site infection, ventilatorassociated pneumonia, and BSI include the following: no cases of ventilator-associated pneumonia since April 2005 at St. Mary's Health Center (Jefferson City, MO); no cases of catheter-related BSI for 15 and 17 months in 2 adult intensive care units (ICUs) and during 2 years in the neonatal ICU of DuBois Regional Medical Center, (DuBois, PA); $75 \%$ reduction in the number of catheter-related BSIs, with an infectionfree interval extending to 359 days, at Beth Israel Medical Center (New York, NY); no cases of ventilator-associated pneumonia in 20 months, and only 2 cases in nearly 3 years, at Dominican Hospital-Catholic Healthcare West (Santa Cruz, CA).$^{10}$ Although no independent attempt was made to verify these voluntary reports, or to assess the extent to which standard case criteria, such as those promulgated by the CDC, were used, these results are impressive enough that they cannot be easily dismissed.

Recently published results from the Keystone Project, which involved 108 ICUs (103 reporting data), primarily in Michigan, demonstrate that collaborative quality improve- ment initiatives can reduce infection rates substantially. The median number of cases of catheter-related BSI per 1,000 catheter-days decreased from 2.7 at baseline to 0 just 3 months after implementation of the intervention. Improvement was sustained, with the mean number of cases per 1,000 catheter-days falling from 7.7 at baseline to 1.4 at follow-up 16-18 months later. ${ }^{11}$ In another collaborative project in southwestern Pennsylvania involving 32 hospitals, the Pittsburgh Regional Health Initiative, in collaboration with the CDC, decreased the rate of catheter-related BSI by $68 \%$ in 69 ICUs over 4 years by promoting interventions similar to those in the 100,000 Lives Campaign's BSI bundle. ${ }^{12}$ In a prospective, single-institution study, catheter-related BSI was virtually eliminated through implementation of a bundle of evidence-based interventions. ${ }^{13}$ Studies such as these represent a breakthrough in rigorous evaluation of quality improvement interventions in infection control-an urgent need, as cited in the recent evidence-based review commissioned by the Agency for Healthcare Research and Quality. ${ }^{14}$

These findings strongly suggest that we have entered a new era of quality improvement in infection control. "Getting to zero" is no longer a wild fantasy, but a possible result of strong will, application of evidence-based practices, and robust execution.

Encouraged by progress in reducing the incidence of infections associated with invasive devices, many hospitals are now turning attention to prevention and control of infection due to HA-MDROs, especially MRSA. Indeed, an intervention to reduce the incidence of MRSA infection is one of the 6 new interventions in the IHI's 5 Million Lives Campaign. Incorporating advice from an expert panel, and with active collaboration and support from the CDC facilitated by an on-site representative (one of us, B.L.Z.) sponsored by the $\mathrm{CDC}$ and the IHI's George W. Merck Fellowship Program, as well as collaboration and support from the Society for Healthcare Epidemiology of America (SHEA) and the Association for Professionals in Infection Control and Epidemiology (APIC), the IHI developed an MRSA intervention package with 5 components: compliance with hand hygiene, decontamination of the environment and equipment, use of active surveillance cultures to identify MRSA reservoirs and track transmission, use of contact precautions for those infected and colonized, and reliable adherence to the central venous catheter and ventilator bundles. ${ }^{15}$

The emphasis on MRSA in the 5 Million Lives Campaign is timely. Encouraged by data from some northern European countries demonstrating sustained very low rates of MRSA colonization and infection, hospitals in the United States, France, the United Kingdom, and other countries where MRSA is more entrenched are mounting aggressive programs to control this pathogen. Although most studies to date have not met the highest standards of epidemiological rigor (eg, cluster-randomized trials or properly conducted interrupted time-series analysis), some institutions have reported promising results. One of the earliest encouraging studies was re- 
ported from Geneva, in which Pittet et al. ${ }^{16}$ noted a sustained reduction in MRSA transmission and HAIs temporally related to a multifaceted hand-hygiene campaign that included bedside placement of alcohol-based hand rub and use of active surveillance cultures. ${ }^{16}$ A recent study from the Brigham and Women's Hospital (Boston, MA) that used an interrupted time-series design is particularly noteworthy. Huang et al. ${ }^{17}$ implemented, in stages, a series of interventions to reduce MRSA transmission in 10 ICUs, including the use of full barrier precautions for placement of central venous catheters, a hand hygiene campaign with hospital-wide promotion of alcohol-based hand rubs, and, finally, the use of active surveillance cultures. The sequential promotion of these measures did not have a sustained impact until the use of active surveillance cultures was added, although compliance with the previous interventions may have been suboptimal. The overall rate of MRSA BSI fell by $75 \%$ in ICUs. Notably, even though the aggressive interventions were confined to the ICUs, there was a $40 \%$ decrease in BSIs due to MRSA over 8 years in non-ICU areas of the hospitals. ${ }^{17}$

Other reports, although less robust epidemiologically, suggest that dramatic improvement is possible. Following on the regional success with BSI prevention in southwest Pennsylvania, the VA Pittsburgh Health System and other hospitals in the region, in collaboration with the $\mathrm{CDC}$, launched an initiative to address control of healthcare-associated MRSA infection. The VA Pittsburgh Health System implemented a bundle of interventions, including use of active surveillance cultures, contact precautions, hand hygiene, and changes to hospital "culture" (by means of briefings on patient care units, leadership involvement, and support of problem solving by front-line healthcare workers and other staff). In 2 patient care units in which these intervention were implemented, the MRSA infection rate decreased by more than $50 \% .{ }^{18}$ At the University of Pittsburgh Medical Center Presbyterian Hospital, a similar intervention in the medical ICU was followed by a $90 \%$ reduction in the rate of healthcare-associated MRSA infection, compared to a $20 \%$ reduction in other ICUs that did not participate in the intervention. ${ }^{19}$

Successful improvement efforts, such as those facilitated by the IHI, emphasize the importance of leadership involvement and organizational alignment. While respecting the importance of involving infection control staff and frontline care givers, the IHI engages the leaders who ultimately are accountable for driving the quality improvement agenda and providing the necessary resources: the hospital's executives and board of trustees. Using what has been learned from high-achieving organizations, the IHI suggests the following steps: establish the mission, vision, and strategy with quality at its core; build the foundation for an effective leadership system with clear aims and accountability at each and every level; guarantee transparent measurement; ensure access to both internal and external ideas; test promising approaches and provide resources to implement those that work; and attend relentlessly to execution by placing quality at the top of the agenda and tying it to the financial bottom line. ${ }^{20}$ This requires moving from exhorting frontline staff to work harder at improvement to making quality and safety core institutional priorities and aligning goals, execution, and measurement at every level of the organization. In addition, it means respecting the role that every stakeholder in the care process (including the patient) has in driving improvement, treating each adverse event as an opportunity to analyze what went wrong and adapting procedures immediately, creating a safety culture that minimizes blame and maximizes systems improvements, communication, and teamwork.

Until recently, HAIs have been seen as an unfortunate but inevitable consequence of health care. This attitude, and the behavior it engenders, are no longer supportable. Although benchmarking may dominate some discussions of mandatory public reporting, hospitals should strive for a culture in which every infection is seen as an opportunity for improvement, and the goal is dramatic improvement with a relentless march towards "zero tolerance." Infection control professionals have the knowledge and expertise to play a critical role in this new paradigm, but only if they see themselves as part of a multidisciplinary team. Some of the greatest successes in recent years have been driven by intensivists and surgeons who were fed up with the status quo and the culture of acceptance and had the heart and nerve to raise the bar. They succeeded in part because infection control professionals seized the moment. At long last, after a half century of pushing their agenda through a labyrinth of indifference, the door is open and the path is clear. In fact, striving for zero, rather than benchmarks, should be our goal. The public and legislators are demanding attention to healthcare quality and patient safety, with specific attention to HAI, through state-mandated public reporting and calls for choice based on transparency. The public is not waiting for perfect measurement or strategies. Given the multitude of success stories that illustrate what is possible, neither should we.

In light of ongoing differences of opinion regarding how best to prevent ever-increasing rates of HA-MDRO transmission, $\mathrm{CDC} / \mathrm{HICPAC}$ has taken a results-oriented approach similar to that of the IHI. Their recently released HA-MDRO guideline does not come down squarely on one side of a heated debate, but rather recommends adopting whatever evidencebased interventions are necessary to reduce infections and save lives. The guideline encourages hospitals to begin with the prevention measures that everyone agrees upon, but be ready to quickly incorporate the more controversial interventions until sustained decreases in HA-MDRO transmission and infection are achieved. ${ }^{21}$ Unlike previous HA-MDRO guidance, the recommendations of $\mathrm{CDC} / \mathrm{HICPAC}$, like those of the IHI and the SHEA/APIC guideline, ${ }^{22}$ now challenge US healthcare facilities to improve outcomes dramatically through reliable implementation of infection control practices. By focusing on results and providing multiple prevention options that healthcare facilities can apply, the CDC guideline allows flexibility in designing a prevention program while requiring facilities to use 
all measures necessary to protect patients against drug-resistant organisms and the potentially lethal infections they cause. As the successes achieved by the IHI and others demonstrate, the time is right to relegate studies describing the burden of these infections to history and make effective prevention the new status quo.

Address reprint requests to Bonnie L. Zell, MD, MS, 20 University Road, 7th floor, Cambridge, MA 02138 (bzell@ihi.org).

\section{REFERENCES}

1. Klevens RM, Edwards JR, Tenover FC, et al. Changes in the epidemiology of methicillin-resistant Staphylococcus aureus in intensive care units in US hospitals, 1992-2003. Clin Infect Dis 2006; 42:389-391.

2. Fabbro-Perray P, Sotto A, Defez C, et al. Mortality attributable to nosocomial infection: a cohort of patients with and without nosocomial infection in a French university hospital. Infect Control Hospital Epidemiol $2007 ; 28: 265-272$ (in this issue).

3. Shurland S, Zhan M, Bradham DD, Roghmann MC. Comparison of mortality risk associated with bacteremia due to methicillin-resistant and methicillin-susceptible Staphylococcus aureus. Infect Control Hospital Epidemiol 2007; 28:273-279 (in this issue).

4. Graves N, Weinhold D, Tong E, et al. Effect of healthcare-acquired infection on length of hospital stay and cost. Infect Control Hospital Epidemiol 2007; $28: 280-292$ (in this issue).

5. Noskin GA, Rubin RJ, Schentag JJ, et al. The burden of Staphylococcus aureus infections on hospitals in the United States: an analysis of the 2000 and 2001 Nationwide Inpatient Sample Database. Arch Intern Med 2005; 165:1756-1761.

6. Stukel TA, Fischer ES, Wennberg DE, Alter DA, Gottlieb DJ, Vermeulen MJ. Analysis of observational studies in the presence of treatment selection bias: effects of invasive cardiac management on AMI survival using propensity score and instrumental variable methods. JAMA 2007; 297:278-285.

7. Berwick DM, Calkins DR, McCannon CJ, Hackbarth AD. The 100,000 Lives campaign: setting a goal and a deadline for improving health care quality. IAMA 2006; 295:324-327.

8. Guidelines for the management of adults with hospital-acquired, ventilator-associated, and healthcare-associated pneumonia. Am J Respir Crit Care Med 2005; 171:388-416.

9. Nolan T, Berwick DM. All-or-none measurement raises the bar on performance. JAMA 2006; 295:1168-1170.

10. Institute for Healthcare Improvement Web site. Available at: http:
//www.ihi.org/IHI/Programs/Campaign/Campaign.htm?TabId $=2 \#$ InterventionMaterials. Accessed January 20, 2007.

11. Pronovost $P$, Needham $D$, Berenholtz $S$, et al. An intervention to decrease catheter-related bloodstream infections in the ICU. $N$ Engl J Med 2006; 355:2725-2732.

12. Reduction in central line-associated bloodstream infections among patients in intensive care units-Pennsylvania, April 2001-March 2005. MMWR Morb Mortal Wkly Rep 2005;54(40):1013-1016.

13. Berenholtz SM, Pronovost P, Lipsett PA, et al. Eliminating catheterrelated bloodstream infections in the intensive care unit. Crit Care Med 2004; 32:2014-2020.

14. Agency for Healthcare Research and Quality. Closing the Quality Gap: A Critical Analysis of Quality Improvement Strategies. Vol. 6. Prevention of Healthcare-Associated Infections. Rockville, MD: Agency for Healthcare Research and Quality: 2007.

15. Institute for Healthcare Improvement. Reduce methicillin-resistant Staphylococcus aureus (MRSA) "how-to guide." 2006. Available at: http://www.ihi.org/IHI/Programs/Campaign/Campaign.htm?TabId =2\# ReduceMethicillin-ResistantStaphylococcusAureus(MRSA)Infection. Accessed February 2, 2007.

16. Pittet D, Hugonnet S, Harbarth S, et al. Effectiveness of a hospital-wide programme to improve compliance with hand hygiene. Infection Control Programme. Lancet 2000; 356:1307-1312.

17. Huang SS, Yokoe DS, Hinrichsen VL, et al. Impact of routine intensive care unit surveillance cultures and resultant barrier precautions on hospital-wide methicillin-resistant Staphylococcus aureus bacteremia. Clin Infect Dis 2006; 43:971-978.

18. Muder R, McCray E, Cunningham $\mathrm{C}$, et al. Sustained reduction in methicillin-resistant Staphylococcus aureus (MRSA) infection following implementation of a systems engineering approach. In: Program and abstracts of the 16th annual meeting of the Society for Healthcare Epidemiology of America; March 18-21, 2006; Chicago, Illinois. Abstract 66.

19. Muto C, Blank MA, Pokrywka M, et al. Reduction of methicillin-resistant Staphylococcus aureus (MRSA) hospital-acquired infections (HAIs) in a medical (M) ICU: effect of a MRSA control (C) initiative (I) during a concurrent central-line associated BSI (CLAB) reduction. In: Program and abstracts of the 16th annual meeting of the Society for Healthcare Epidemiology of America; March 18-21, 2006; Chicago, Illinois. Abstract 67.

20. Reinertsen JL, Pugh MD, Bisognano M. Seven Leadership Leverage Points for Organization-Level Improvement in Health Care. Cambridge, MA: Institute for Healthcare Improvement; 2005.

21. Siegel J, Rhinehart E, Jackson M, Chiarello L. Management of multidrugresistant organisms in healthcare settings, 2006. Atlanta, GA: Centers for Disease Control Healthcare Infection Control Practices Advisory Committee; 2006.

22. Muto CA, Jernigan JA, Ostrowsky BE, et al. SHEA guideline for preventing nosocomial transmission of multidrug-resistant strains of Staphylococcus aureus and Enterococcus. Infect Control Hosp Epidemiol 2003; 24:362-386. 